AIAA 2001-2527

\title{
Simultaneous Aerodynamic and Structural Design Optimization (SASDO) for a 3-D Wing
}

\author{
Clyde R. Gumbert \\ NASA Langley Research Center \\ Hampton, Virginia \\ Gene J.-W. Hou \\ Old Dominion University \\ Norfolk, Virginia \\ Perry A. Newman \\ NASA Langley Research Center \\ Hampton, Virginia
}

preprint of the paper presented at the

AIAA $15^{\text {th }}$ Computational Fluid Dynamics Conference

June 11-14, 2001

Anaheim, CA 
AIAA 2001-2527

\title{
Simultaneous Aerodynamic and Structural Design Optimization (SASDO) for a 3-D Wing
}

\author{
Clyde R. Giumbert* \\ NASA Langley Research Center. Hampton. IA 23681 \\ Gene .J. -W. Hou ${ }^{\dagger}$ \\ Old Dominion University. Norfolk, IA 23599-0247 \\ Perry A. Newman ${ }^{\ddagger}$ \\ NASA Langley Research Center. Hampton. IA 23681
}

\begin{abstract}
The formulation and implementation of an optimization method called Simultaneous Aerodynamic and Structural Design Optimization (SASDO) is shown as an extension of the Simultaneous Aerodynamic Analysis and Design Optimization (SAADO) method. It is extended by the inclusion of structure element sizing parameters as design variables and Finite Element Method (FEM) analysis responses as constraints. The method aims to reduce the computational expense incurred in performing shape and sizing optimization using state-of-the-art Computational Fluid Dynamics (CFD) flow analysis, FEM structural analysis and sensitivity analysis tools. SASDO is applied to a simple, isolated, 3-D wing in inviscid flow. Results show that the method finds the same local optimum as a conventional optimization method with some reduction in the computational cost and without significant modifications to the analysis tools.
\end{abstract}

\section{Nomenclature}

$b$

$C_{D}$

$C_{L}$

$C_{m}$

$c_{r}$

$c_{t}$

F

$g$

$\pi$

$L$

$M_{\infty}$

$n$

$p$

$P$

$q \alpha$

$Q$

wing semispan

drag coefficient

lift coefficient

pitching moment coefficient

wing root chord

wing tip chord

design objective function

design constraints

stiffness matrix

aerodynamic loads

free-stream Mach number

unit normal vector

local aerodynamic pressure compliance. the work done by the aerodynamic load to deflect the structure

free-stream dynamic pressure

flow-field variables (state variables) at each (FD mesh point

*Research Engineer. Multidisciplinary Optimization Branch M/S 159. c.l.gumbert ilarc.nasa.gov

tProfessor, Department of Mechanical Engineering, ALAA member, ghouglions.odu.edu

ISenior Research Scientist. Multidisciplinary Optimization Branch, M/S 159, p.a.newmancularc.nasa.gov

Copyright 2001 by the American Institute of Aeronautics and Astronautics. Inc No copyright is asserted in the Inited States under Title 17 . U.S. Code' The I'S. Government has a rovaltyfree license to exircise all rights under the copyright clamed herein for Governmental Purposes. All other rights are reserved by the copyright owner.
$\Delta Q_{1} \quad$ change in flow solver field variables due to better analysis convergence

$\Delta Q_{2} \quad$ change in flow solver field variables due to design changes

$R \quad$ aerodynamic state equation residuals at each CFD mesh point

$\left|R / R_{0}\right|$ norm of the residual ratio, current/initial

$s \quad$ surface area

$S \quad$ semispan wing planform area

$u \quad$ structural deflections (state variables)

$\Delta u_{1} \quad$ change in deflections due to better analysis convergence

$\Delta u_{2} \quad$ change in deflections due to design changes

W wing weight

X CFD volume mesh coordinates

$x_{L E} \quad$ location of wing root leading edge

$r \quad$ chordwise location normalized by local wing section chord

$x_{t} \quad$ longitudinal location of wing tip trailing edge

root section maximum camber

free-stream angle-of-attack

design variables

structural element size factor

line search parameter

operator which indicates a change in a variable 


$\begin{array}{ll}\varepsilon, \varepsilon^{\prime} & \text { convergence tolerances for function and gra- } \\ & \text { dients } \\ \lambda & \text { adjoint variablo for } Q \\ \mu & \text { adjoint variable for } u \\ \tau & \text { twist angle at wing tip, positive for leading } \\ & \text { edge up }\end{array}$

\section{Subscripts}

d deflected shape

$j \quad$ jig (undeflected) shape

$s \quad$ wing surface mesh

$v \quad$ wing volume mesh

\section{Superscripts}

* designates updated value

' gradient with respect to design variables

\section{Introduction}

Simultaneous Aerodynamic Analysis and Design Optinization (SAADO) is a procedure that incorporates design improvement within the iteratively solved (nonlinear) aerodynamic analysis so as to achieve fully converged flow solutions only near an optimal design. When $S A A D O$ is applied to a flexible wing rather than a rigid wing, the linear Finite Element Method (FEM) solution is iteratively coupled with the nonlinear (Computational Fluid Dynamics (CFD) solution. When design variables that control structural element size are included, it is renamed Simult aneous Aerodynamic and Structural Design Optimization (SASDO). Overall computational efficiency is achieved because the many expensive iterative (nonlinear) solutions for non-optimal design parameters are not converged (i.e., obtained) at each optimization step. One can obtain the design in the equivalent of a few (rather than many) multiples of the computational time for a single, fully converged coupled aero-structural analysis. SAADO and similar procedures for simultaneous analysis and design (SAND) developed by others are noted and discussed by Newman et al. ${ }^{1}$ These SANI) procedures appear best suited for applications where the discipline analyses involved in the design are nonlinear and solved iteratively. Generally, convergence of these discipline analyses (i.e., state equations) is viewed as an equality constraint in an optimization problem. From this latter point of view the SASDO method proceeds through infeasible regions of the design space which includes not only the design variables 3. but also the state variables $Q$ and $u$. A further advantage of $S A S D O$ is the efficient utilization of existing discipline analysis codes (without internal changes). augmented with sensitivity or gradient information, and yet effectively coupled more tightly than is done in conventional gradient-based optimization procedures, referred to as nested analysis and design (NAND) procedures. ${ }^{1}$ A recent overview of aerodynamic shape optimization $^{2}$ discusses both NAND and SAND procedures in the context of current steady aerodynanic optimization research.

For single-discipline design problems, the distinction between NAND and SAND procedures is fairly clear and readily seen. With respect to discipline feasibility (i.e., convergence of the generally nonlinear, iteratively solved state equations), these procedures (an be viewed as accomplishing design by using only very well converged discipline solutions (NAND), or as converging a sequence of discipline solutions from poorly to well as the design progresses (SAND). However. the problem formulation and solution algorithms may differ considerably. About twenty SAND references are quoted by Newman et al. ${ }^{1}$ and Newman et al. ${ }^{2}$ these references discuss a variety of formulations, algorithms, and results for single-discipline problems (mostly CFD applications) in the sense of SAND as defined above. For mult idisciplinary design optimization problens, the distinction between NAND and SAND is somewhat blurred because there are feasibility considerations with respect to all the individual discipline state equations, as well as with respect to the multidisciplinary system compatibility and constraints. A number of the papers in Ref. 3 discuss MDO formulations and algorithms that are called SAND-like; however, not all of these latter MDO procedures appear to agree with the sense of $S A N D$ defined above and used herein; one that does is Ref. 4.

The computational feasibility of SAADO for quasi 1-D nozzle shape design based on the Euler equation (Fl) approximation was demonstrated by Hou et al. ${ }^{5}$ and Mani. "Application of SAADO for turbulent transonic airfoil shape design based on a 2-D thin-layer Navier-Stokes (FD) approximation was demonstrated and reported in a later paper by Hou et al. ${ }^{7}$ Both of these application results are summarized and briefly discussed in Ref. 1. The application of SAADO for rigid 3-D wing clesign based on the Euler (FD approximation was presented in Ref. 8 . These SAADO procedures utilized quasi-analytical sensitivity derivatives obtained from hand-differentiated code for the initial quasi 1-D application ${ }^{5, t}$ and from automatically differentiated code for both the 2-1) airfoil application $^{\top}$ and the $3-\mathrm{D}$ rigid wing application. ${ }^{8}$ Different optimization techniques have also been used in these SAADO procedures.

The extension to multidisciplinary analysis with shape design variables only was presented in Ref. 9. Our initial results from SASDO are given in this paper. The analysis problem, the objective function, and the constraints are the same as those used in Ref. 9. That is, changes in design variables are sought to produce improvenent in the lift-to-drag ratio of a simple wing subject to both aerodynamic and structural solution-dependent constraints. These constraints are the difference between the lift and weight, the pitch- 
ing moment coefficient, and the compliance, a function representing work done by the aerodynamic load to deflect the structure. There are also geometric constraints.

The flexible wing studied here is formulated as a static aeroelastic problem. Similar problens have been used as examples in Refs. 10-15 to study various solution strategies for multidisciplinary analysis and optimization. In particular, Arian ${ }^{12}$ analyzed the Hessian matrix of the system equations to derive the mathematical conditions under which the aeroelastic optimization problem can be solved in a "loosely" coupled manner. The multidisciplinary research of Walsh et al. ${ }^{13.14}$ emphasized engineering aspects of integrating high fidelity disciplinary analysis software and distributed computing over a network of heterogeneous computers. The aeroelastic analysis results of Reuther et al. ${ }^{15}$ were verified with experimental data.

Only a limited amount of literature related to aeroelastic problems has elaborated on the coupled sensitivity analysis. Kapania, Eldred and Barthelemy; ${ }^{\text {to }}$ Arslan and (arlson: ${ }^{17}$ and (Giunt a and SobieszczanskiSobieski ${ }^{18}$ derived global sensitivity equations (GSEs); some matrix coefficients in these GSEs were evaluated by finite differencing. Cruinta ${ }^{1 ! \prime}$ later introduced modal coordinates to approximate the elastic displacement vector in order to reduce the size of the CSSE. Newnan, Whitfield, and Anderson ${ }^{20}$ used the complex variable approach to obtain the aeroelastic sensitivity derivatives, whereas Reuther et al. ${ }^{15}$ employed the adjoint variable approach to derive the aeroelastic sensitivity expations. A mathematical study of the coupled nonlinear, incompressible aeroelastic analysis and sensitivity analysis problems has been given by Ghattas and $\mathrm{Li}^{21}$ Recent results on aeroelastic sensitivity analysis and optimization can be found in Refs. 22-24. Particularly, Maute et al. ${ }^{23}$ and $\mathrm{Hou}$ and Satyanarayana ${ }^{24}$ explicitly formulated the deflection update and the load transfer between the separate flow and structures solvers as part of the coupled sensitivity equations. In the present study, the coupled sensitivity equations are constructed by differentiating the aeroelastic state equations and solving them by a (ieneralized Gauss-Seidel (GGS) method. ${ }^{12}$ The present SASDO concept is very similar to that of Ghat as and others, Refs. $4,21,25,20$, but differs in the implementation details as described later.

\section{Problem Description}

To evaluate the efficacy of the SASDO procedure for a problem involving multidisciplinary analysis, it is applied herein to a simple, isolated, flexible wing. The wing shape consisted of a trapezoidal planform with a rounded tip. It was parameterized by fifteen variables; five described the planform, and five each described the root and tip section shapes. A schematic of the wing and its associated shape parameters is shown in
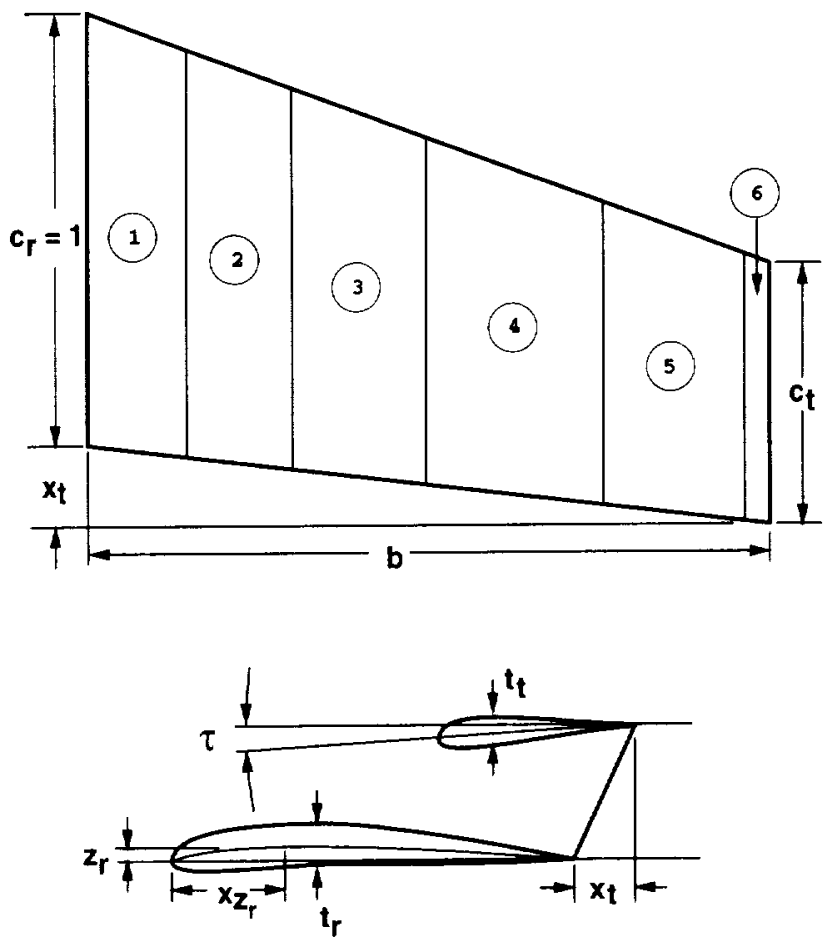

Fig. 1 Description of semispan wing parameterization.

Fig. 1. The baseline wing section varied linearly from an NAC A 0012 at the root to an NACA 0008 at the tip. The wing structure consists of a skin, ribs, and spars. The ribs and spars consist of shear webs and trusses. Six spanwise zones of the structural model are defined as depicted in Fig. 1. The relative sizes of the skin and web thicknesses and the truss ross section areas are fixed within each zone. Each zone is assigned a parameter $\Gamma$ which multiplies all the thicknesses and areas of the structural elenents in that zone. The specific parameters selected as design variables in the sample optimization problems are identified in the section entitled Results. The objective function to be minimized was the negative of the lift-to-drag ratio, $-L / D$. Both coupled solution-dependent and geometric constraints were inposed.

The solution-dependent constraints were

- lower linit on the difference between the total lift and the structural weight, $\left(C_{L} * S * q x-11\right.$

- upper limit on compliance, $P=\oint p u \cdot n d s$

- upper limit on pitching moment, $C_{m}$, in lieu of a trim const raint

The purely geometric constraints were

- minimum leading edge radius, in lieu of a manufacturing requirement.

- side constraints (bounds) on the active design variables 


\section{SASDO Procedure}

\section{Formulation}

The flexible SASDO approach formulates the design optimization problem as follows:

$$
\min _{\beta, Q, u} F\left(Q . \mathrm{X}_{d u^{\prime}}(\beta, u), u, \beta\right)
$$

subject to

$$
y_{i}\left(Q, K_{t u}(3, u), u, 3\right) \leq 0 ; \quad i=1,2 \ldots, m
$$

where the flow ficld $Q$ and the structural deflection $\mathrm{u}$ are solutions of the coupled flow equation

$$
R\left(Q . X_{d,}(3, u), 3\right)=0
$$

and the finite element structural equation

$$
K\left(X_{j}(\beta), \beta\right) u=L\left(Q, X_{t u}(\beta, u)\right)
$$

The deflected volume mesh, $\mathrm{V}_{t}$, is determined by the deflected surface mesh, $X_{d s}$, as $X_{d s}=X_{d}\left(X_{d s}\right)$. The deflected surface mesh is a result of the jig shape augmented by the elastic deflortion. 1 . as $\mathrm{K}_{\mathrm{ds}}=$ $\mathrm{S}_{j}(3)+u$. The two disciplines are coupled through the deflection, $u$, and the load, $L$.

Recall that $Q, R$, and $S_{d v^{\prime}}$ are very large vectors This formulation treats the state variables, $Q$ and $u$, as part of the set of independent design variables, and consiclers the state equations to be constraints. Becalse satisfaction of the equality constraints, Eqs. (3) and (4), is required only at the final optimum solution, the coupled steady-state aero-structural field equations are not converged at every design-optimization iteration. The easing of that restriction is expected to significantly reduce the excessively large computational cost incurred in the conventional approach. However, this advantage would likely be offset by the very large increase in the number of design variables and equality ronstraint functions, unless some remedial procedure is adopted.

\section{Approximations}

The SASIOO method begins with a hinearized design optimization problem solved for the most favorable change in the design variables, $\Delta ;$, as well as for the changes in the state variables, $\Delta Q$ and $\Delta u$ : that is,

$$
\begin{array}{rl}
\min _{\Delta x, \Delta Q, \Delta u} & F\left(Q, X_{d u}(\beta, u), u, \beta\right) \\
& +\frac{\partial F}{\partial Q} \Delta Q+\left(\frac{\partial F}{\partial u}+\frac{\partial F}{\partial X_{d v}} \frac{\partial X_{d u}}{\partial \dot{\lambda}_{d s}}\right) \Delta u \\
& +\left(\frac{\partial F}{\partial \boldsymbol{X}_{\mathrm{d} t}} \frac{\partial \boldsymbol{X}_{d u}}{\partial \boldsymbol{X}_{u}} \Gamma_{j}^{\prime}+\frac{\partial F}{\partial \beta}\right) \Delta \beta
\end{array}
$$

subject to inequality constraints

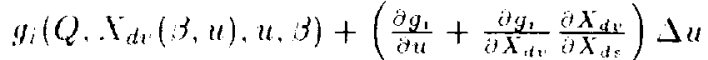

$$
\begin{aligned}
& +\frac{\partial g_{1}}{\partial Q} \Delta Q+\left(\frac{\partial g_{2}}{\partial \boldsymbol{X}_{d}} \frac{\partial \boldsymbol{\lambda}_{d v}}{\partial \boldsymbol{X}_{d}} \mathrm{Y}_{j}^{\prime}+\frac{\partial g_{2}}{\partial \beta}\right) \Delta \beta \leq 0 ; \\
& i=1.2 \ldots . m
\end{aligned}
$$

and equality constraints

$$
\begin{aligned}
& R\left(Q, X_{d v}(\beta, u), \beta\right)+\frac{\partial R}{\partial Q} \Delta Q+\frac{\partial R}{\partial \boldsymbol{X}_{d v}} \frac{\partial X_{d v}}{\partial \boldsymbol{X}_{d s}} \Delta u \\
& +\left(\frac{\partial R}{\partial \boldsymbol{X}_{d v}} \frac{\partial \boldsymbol{X}_{d v}}{\partial \boldsymbol{X}_{d s}} X_{j}^{\prime}+\frac{\partial R}{\partial, \beta}\right) \Delta \beta=0
\end{aligned}
$$

and

$$
\begin{aligned}
& h\left(X_{j}(\beta), \beta\right) u-L\left(Q, X_{d u}(\beta, u)\right)-\frac{\partial L}{\partial Q} \Delta Q \\
& +\left(K-\frac{\partial I}{\partial X_{d r}} \frac{\partial X_{d v}}{\partial X_{d s}}\right) \Delta u+\left(\frac{\partial K}{\partial \beta} u\right) \Delta \beta \\
& +\left(\frac{\partial K}{\partial X_{j}} u-\frac{\partial L}{\partial X_{d t}} \frac{\partial \lambda_{d v}}{\partial X_{d s}}\right) X_{j}^{\prime} \Delta \beta=0
\end{aligned}
$$

Note that Eqs. (5) through (8) are linearized approximations of Eqs. (1) through (4), respectively.

In this formulation, neither the residual of the nonlinear aerodynamic field equations, $R(Q \ldots \mathrm{X}, \beta)$. nor that of the structures equation. $K u-L$, is required to be zero (reach target) until the final optimum design is achieved. The linearized problem of Eqs. (5) through $(8)$ is difficult to solve directly because of the number of design variables and equality constraint equations.

\section{Direct differentiation method}

One way to overcome this difficulty is by the direct differentiation method. In this method $\Delta Q, \Delta u$, and Eqs. $(7)$ and $(8)$ are removed altogether from the linearized problem by direct substitution. This is achieved by expressing $\Delta Q$ and $\Delta u$ as functions of $\Delta 3$.

$$
\begin{aligned}
\Delta Q & =\Delta Q_{1}+\Delta Q_{2} \Delta \beta \\
\Delta u & =\Delta u_{1}+\Delta u_{2} \Delta \beta
\end{aligned}
$$

where vectors $\Delta Q_{1}$ and $\Delta u_{1}$ are corrections in the aeroelastic solution due to the improvement of coupled aeroelastic analysis, while matrices $\Delta Q_{2}$ and $\Delta u_{2}$ are corrections due to changes in the design variables. These vectors and matrices are solutions of the following coupled sets of equations, obtained from Eqs. (7) and $(8)$ :

$$
\begin{aligned}
& \frac{\partial R}{\partial Q} \Delta Q_{1}=-R-\frac{\partial R}{\partial \lambda_{d v}} \frac{\partial \lambda_{d v}}{\partial X_{d s}} \Delta u_{1} \\
& \kappa \Delta u_{1}=\frac{\partial L}{\partial Q} \Delta Q_{1}+\frac{\partial L}{\partial \lambda_{d t}} \frac{\partial \lambda_{d u}}{\partial \lambda_{d s}} \Delta u_{1}
\end{aligned}
$$

where, for the linear FEM, $L u-L=0$ at every iteration, and

$$
\begin{aligned}
& \frac{\partial R}{\partial Q} \Delta Q_{2}+\frac{\partial R}{\partial X_{d v}} \frac{\partial X_{d u}}{\partial X_{d s}}\left(X_{j}^{\prime}+\Delta u_{2}\right)+\frac{\partial R}{\partial j}=0 \\
& K \Delta u_{2}=\frac{\partial L}{\partial Q} \Delta Q_{2}+\frac{\partial L}{\partial X_{d \prime}} \frac{\partial X_{d u}}{\partial X_{d s}}\left(Y_{j}^{\prime}+\Delta u_{2}\right) \\
& -\left(\frac{\partial K}{\partial X_{1}} \cdot Y_{j}^{\prime}+\frac{\partial K}{\partial g}\right) u
\end{aligned}
$$

Note that the number of columns of matrices $\Delta Q$ and $\Delta u_{2}$ is equal to the number of design variables. $\beta$. Thus the computational cost of Eq. (11) is directly proportional to the number of design variables.

A now linearized problem with $\Delta / 3$ as the only design variables can be obtained by substituting Eq. (9) into 
Eqs. (5) and (6) for $\Delta Q$ and $\Delta u$.

$$
\begin{array}{ll}
\min _{\Delta \beta} & F(Q, X, u, \beta)+\frac{\partial F}{\partial Q} \Delta Q_{1} \\
+ & \left(\frac{\partial F}{\partial u}+\frac{\partial F}{\partial X_{d t^{\prime}}} \frac{\partial X_{d v}}{\partial X_{d s}}\right) \Delta u_{1} \\
+ & \left\{\frac{\partial F}{\partial Q} \Delta Q_{2}+\frac{\partial F}{\partial X_{d v}} \frac{\partial X_{d v}}{\partial X_{d s}} X_{j}^{\prime}\right. \\
& \left.+\left(\frac{\partial F}{\partial X_{d v}} \frac{\partial X_{d v}}{\partial X_{d s}}+\frac{\partial F}{\partial u}\right) \Delta u_{2}+\frac{\partial F}{\partial \beta}\right\} \Delta \beta
\end{array}
$$

subject to

$$
\begin{aligned}
& g_{i}(Q, \mathrm{X}, u, \beta)+\frac{\partial g_{1}}{\partial Q} \Delta Q_{1} \\
& +\left(\frac{\partial g_{1}}{\partial u}+\frac{\partial g_{1}}{\partial X_{d v}} \frac{\partial X_{d v}}{\partial X_{d s}}\right) \Delta u_{1} \\
& +\left\{\frac{\partial g_{1}}{\partial Q} \Delta Q_{2}+\frac{\partial g_{1}}{\partial X_{d v}} \frac{\partial X_{d u}}{\partial X_{d s}}\left(X_{j}^{\prime}+\Delta u_{2}\right)\right. \\
& \left.\quad+\frac{\partial g_{1}}{\partial u} \Delta u_{2}+\frac{\partial g_{1}}{\partial \beta}\right\} \Delta \beta \leq 0 ; \quad i=1,2, \ldots, m
\end{aligned}
$$

The appearance of $\Delta Q_{1}$ and $\Delta u_{1}$ in the formulation indicates the difference between the SASDO (SAND) method and the conventional (NAND) aerodynamic optimization method. The $\Delta Q_{1}$ and $\Delta u_{1}$ not only constitute a change in $Q$ and $u$, but also play an inportant role in defining the objective function of Eq. (12) and the constraint violation of Eq. (13). We can directly solve Eqs. 10 for $\Delta Q_{1}$ and $\Delta u_{1}$, and, in fact, in previous $\mathrm{SAADO}$ applications that is how $\Delta Q_{1}$ was determined. However, since $\Delta Q_{1}$ and $\Delta u_{1}$, as shown in Eq. (10), represent a single Newton's iteration on the coupled flow and structures equations, it is possible and less comput ationally expensive to approximate their infuence on the solution $Q$ and $u$ by several Newton's iterations of the coupled aeroelastic equations. That is, $\Delta Q_{1}$ and $\Delta u_{1}$ are not determined explicitly, but rather the first three terms of Eqs. 12 and 13 are viewed as updated values of $F$ and $g_{i}$. Note that the terms in parentheses in Eqs. (12) and (13) are approximated gradients of the objective and constraint functions. Once established, this linearized problem can be solved using any mathematical programming technique for design changes, $\Delta B$. Results presented in this paper are computed using this direct differentiation approach.

\section{Adjoint method}

An alternative way to remove $\Delta Q$ and $\Delta u$ from the linearized problems, Eqs. (5) and (6), is the adjoint variable method. The adjoint variables, $\lambda$ and $\mu$, can be introduced as the solutions of

$$
\begin{aligned}
& \left(\frac{\partial R}{\partial Q}\right)^{T} \lambda=\left(\frac{\partial L}{\partial Q}\right)^{T} \mu-\left(\frac{\partial F}{\partial Q}\right)^{T} \\
& \kappa \mu=\left(\frac{\partial L}{\partial \lambda_{d v}} \frac{\partial \boldsymbol{X}_{d \mu}}{\partial \boldsymbol{X}_{\text {ds }}}\right)^{T} \mu \\
& -\left(\frac{\partial R}{\partial \lambda_{d t}} \frac{\partial \lambda_{d v}}{\partial \lambda_{d s}}\right)^{T} \lambda-\left(\frac{\partial F}{\partial \lambda_{d v}} \frac{\partial \lambda_{d v}}{\partial \lambda_{d s}}\right)^{T}-\frac{\partial F}{\partial u}
\end{aligned}
$$

so as to rewrite the expression of the objective function in $\mathrm{Eq} .(5)$ in terms of $\lambda, \mu$, and $\Delta \beta$ as

$$
\begin{aligned}
& F\left(Q, X_{d v}(\beta, u), u, \beta\right)+\lambda^{T} R+\mu^{T}(K u-L) \\
& +\left\{\left(\frac{\partial F}{\partial X_{d v}} \frac{\partial X_{d v}}{\partial X_{d s}} X_{j}^{\prime}+\frac{\partial F}{\partial \beta}\right)\right. \\
& +\lambda^{T}\left(\frac{\partial R}{\partial X_{d v}} \frac{\partial X_{d v}}{\partial X_{d s}} X_{j}^{\prime}+\frac{\partial R}{\partial \beta}\right) \\
& \left.+\mu^{T}\left(\frac{\partial K^{\prime}}{\partial \lambda^{\beta}} u+\left(\frac{\partial K^{\prime}}{\partial X_{j}} u-\frac{\partial L}{\partial X_{d v}} \frac{\partial X_{d v}}{\partial X_{d s}}\right) \cdot X_{j}^{\prime}\right)\right\} \Delta \beta
\end{aligned}
$$

Note that the terms in the brace represent the gradient of the objective function. The terms $\lambda^{T} R$ and $\mu^{T}(\kappa u-L)$ indicate the effect on the design optimization formulation due to errors in the aeroelastic analysis. Furthermore, in the linear sense, the adjoint variables and the solution errors can be related by the following equations:

$$
\frac{\partial F}{\partial \lambda}=R^{T}
$$

and

$$
\frac{\partial F}{\partial \mu}=(\kappa u-L)^{T}
$$

These equations have been mentioned, for example. by Pierce and Giles ${ }^{27}$ and Venditti and Darmofal ${ }^{28}$ for aerodynamic problems.

In the typical optimization problem there are many design variables. When one can also pose the optimization problem such that there are only a few output quantities for objectives and constraints or. in the extreme, combine the constraints and objective function into a single cost function, the adjoint approach to sensitivity analysis has the advantage that the adjoint solutions are independent of the number of design variables. ${ }^{29}$ However, when the disciplines are loosely coupled, this approach is impractical since the coupled sensitivity analyses would require an adjoint for each disciplinary output being transferred, i.e., the discretized loads and deflections. In a tightly or implicitly coupled multidisciplinary analysis the adjoint approach may prove practical since this system is analogous to a single discipline.

\section{Line Search}

A one-dimensional search on the step size parameter $\gamma$ is then performed in order to find the updated values of $\Delta \beta, \Delta \mathrm{X}, \Delta Q$, and $\Delta u$. Given the search direction $\Delta \beta$ determined by either the direct differentiation method (Eqs. 12 and 1:3) or the adjoint method equivalent, this line search functions to adjust its magnitude so as to simultaneously ensure better results for both design and analysis (converged solutions). The step size parameter $\gamma$ plays the role of a relaxation factor in the standard Newton's iteration. The search procedure employed solves a nonlinear optimization problem of the form

$$
\min _{\gamma} F\left(Q^{*}, Y^{*}, u^{*}, \beta^{*}\right)
$$


subject to

$$
\begin{gathered}
g_{i}\left(Q^{*}, \mathrm{I}^{*}, \mathrm{u}^{*}, \beta^{*}\right) \leq 0 ; \quad i=1,2, \ldots, m \\
R\left(Q^{*}, \mathrm{I}^{*}, \beta^{*}\right)=0
\end{gathered}
$$

and

$$
\Lambda\left(\mathrm{K}^{*}, \beta^{*}\right) u^{*}=L\left(Q^{*}, \mathrm{~K}^{*}\right)
$$

where step size $\gamma$ is the only design variable. Again it is noted for emphasis that the equality constraints. Eqs. (20) and (21), are not required to be zero (reach target) until the final optimum design; violations of these equality const raints must simply be progressively reduced until the $\mathrm{SASDO}$ procedure converges.

The updated $Q^{*}$ and $u^{*}$ can be viewed as $Q^{*}=Q+$ $\Delta Q$ and $u^{*}=u+\Delta u$. where $\Delta Q$ and $\Delta u$ satisfy the first order approximations to E.gs. $(20)$ and (21). That is, $\Delta Q$ and $\Delta u$ are the solutions of Eqs. $(\bar{T})$ and $(8)$ where, in Eq. (9). $\Delta 3$ is replaced by $\Delta \beta^{*}=\gamma \Delta 3$. Consequently, $Q^{*}=Q+\Delta Q_{1}+\gamma \Delta Q_{2} \Delta \beta$ and $u^{*}=$ $u+\Delta u_{1}+\gamma \Delta u_{2} \Delta 3$ are readily available once $?$ is found. The $\Delta()_{1}$ terms appearing in the above SASDO formulation are due to better convergence of the coupled analysis, whereas the $\Delta()_{2}$ terms are due to changes in the design variables. In fact, $\Delta Q_{2}$ and $\Delta u_{2}$ approach the flow field and deflection sensitivities. $Q^{\prime}$ and $u^{\prime}$, as the solution becomes better converged.

\section{Implementation}

The following pseudocode shows algorithuncally how the method was implenented.

set initial analysis convergence tolerance.

set initial solution vectors, $Q$ and $u$.

set initial design variables, $\beta$

do until ronverged

1. solve coupled aeroelastic analysis, Eqs. (3) \& (4), partially converged to $=$

2. compute $F$ and $g$

3. solve conpled aeroelastic sensitivity analysis. Eq. (11), partially converged to $\Xi^{\prime}$

4. compute $\triangle \beta$ terms of Eqs. (5) \& (6)

5. solve optimization problem Eqs. (5) \& (6) for $\Delta \beta$

6. solve Eqs. (18) through (21) for line search parameter,

7. update $\beta, u$, and $Q$

8 . tighten analysis convergence tolerance, $\varepsilon=\varepsilon * \mathcal{Z}$, $0<Z<1$

enddo

This pseudocode is similar to that used in the Biros and Ghattas ${ }^{25}$ SAND approach. Specifically, both approaches use a Sequential Quadratic Programming (SQP) method to solve the design equations (step 5) and an approximate factorization method to solve the system equations (step 1). Step 3 above uses an incremental iterative method with approximate factorization to solve for derivatives in direct mode rather than as a solution of the adjoint equation of

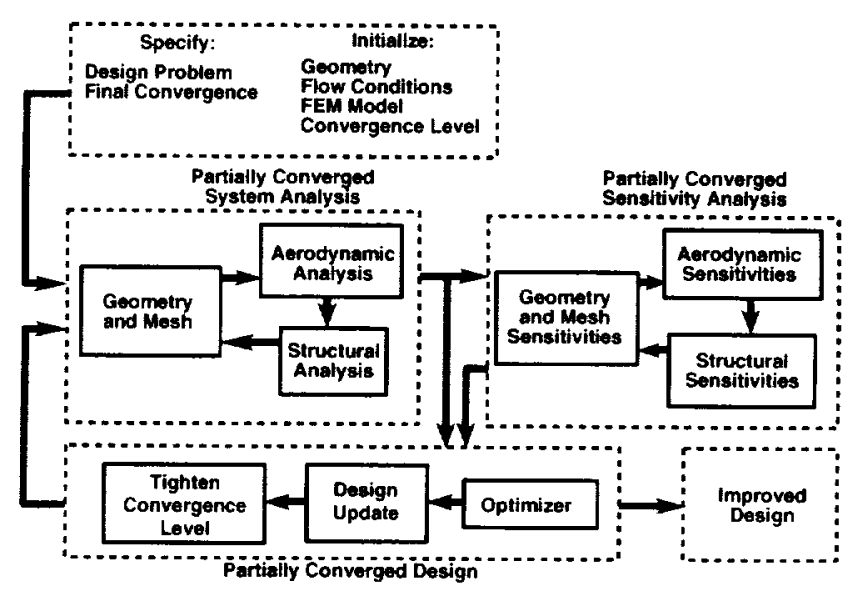

Fig. 2 Diagram of SASDO procedure.

Biros and Ghattas. ${ }^{25}$ In addition, the line search step (step 6) and the convergence tightening step (step 8) were not included in the Biros and (ihattas method. A schematic of the present SASDO procedure is shown in Fig. 2. The dashed box, labeled "Partially Converged System Analyis," depicts the coupled analysis iteration loop. Steps 1 and 2 of the pseudocode: that labeled "Partially Convorged Sensitivity Analysis" depicts the coupled derivative iteration loop. Step 3: that labeled "Partially (onverged Design" depicts the design steps. Steps $\bar{y}-\overline{8}$ of the pseudocode. Specific computational took and methods used to perform the task's depicted by the solid boxes in Fig. 2 are identified in the next sertion.

\section{Computational Tools and Models}

Major computations in this SASI)O procedure are performed using a collection of existing codes. These codes are executed by a separate driver code and scripts that implement the SASDO procedure as just discussed. Each code runs independently, some simultaneously, on different processors. and the required 1/O transfers between them, also directed by the driver, are accomplished via data files.

The aerodynamic flow analysis code used for this study is a version of the CFL3D code 30 Only Euler analyses are performed for this work, although the code is capable of solving the Navier-Stokes equations with any of several turbulence models. The gradient version of this code, whirh was used for aerodynamic sensitivity analysis, was generated by an unconventional application ${ }^{31}$ of the automatic differentiation code $\mathrm{ADIFOR}^{32,33}$ to produce a relatively efficient, direct mode, gradient analysis code. (FL.3D.ADII. ${ }^{34}$ It should be pointed out that the ADIFOR process produces a discretized derivative code consistent with the discretized function analysis code. The addition of a stopping criterion based on the norm of the residual of the field equations was the only modification of the ('FL3D.ADIl code made to accommodate the SASDO procedure. 


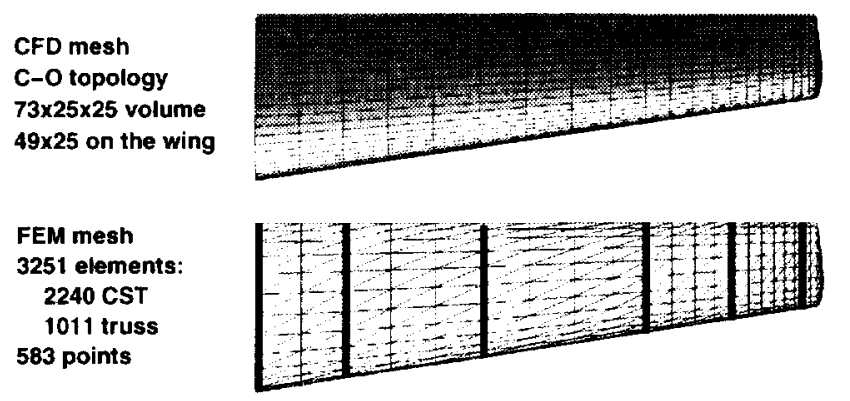

Fig. 3 Computational meshes for wing analysis.

The surface geometry was generated based on the parameters described in a previous section by a code utilizing the Rapid Aircraft Parameterization Input Design (RAPID) technique developed by Smith, et al. ${ }^{35}$ This code was preprocessed with ADIFOR to generate a code capable of producing sensitivity derivatives as well.

The CFD volume mesh needed by the flow analysis code was generated using a version of the CSCMDO ${ }^{30}$ grid generation code. The associated grid sensitivity derivatives needed by the flow sensitivity analysis were generated with an automatically differentiated version of CSCMDO ${ }^{37}$ In addition to the parameterized surface mesh and accompanying gradients. CSCMDO requires a baseline volume mesh of similar shape and identical topology. The 45,000 grid point baseline volume mesh of $\mathrm{C}-\mathrm{O}$ topology used in the present flexible wing examples was obtained with the Gridgen ${ }^{\mathrm{TM}}$ code. The wing surface portion of the mesh is shown in Fig. 3. This mesh is admittedly particularly coarse by current CFD analysis standards.

The structural analysis code $e^{38}$ used to compute the deflection of the elastic wing was a generic finite element code. The flexible structure for the wing shown in Fig. 3 was discretized by 583 nodes; there were 2,141 constant-strain triangle (CST) elements and 1.110 truss elements. Zone boundaries for the design variables controlling element size are also shown in Fig. 3. Because the elastic deformation was assumed to be small, linear elasticity was deemed to be appropriate. The structural sensitivity equations were derived based upon the direct differentiation method. We note that the sensitivity of the aerodynamic forces appears as a term on the right-hand side (RHS) of the deflection sensitivity equations. The derivative of the stiffness matrix in these sensitivity equations was also generated $^{34}$ by using the ADIFOR $^{32,33}$ technique. We note that the coefficient matrix of the structural sensitivity equations was identical to that of the structural equations. Consequently, these structural sensitivity equations were solved efficiently by backward substitution with different RHSs for each sensitivity.

At the wing surface, i.e., the interface where aerodynamic load and structural deflection information is transferred. it was assumed that surface nodes of the
FEM structural model were a subset of the CFD aerodynamic surface mesh points (see Fig. 3) for the present SASDO application. This lack of generality allowed for simplifications in the data transfers and, although an important issue, it was not deemed crucial for these initial SASDO demonstrations. Future applications to more complex configurations should allow for transfer of conserved information between arbit rary meshes as required by the individual disciplines. A recent review of such data transfer techniques and a specific proposed one are given in Ref. 40.

Conventional (NAND) and SASDO (SAND) procedures were implemented using the SQP method of the DOT $^{41}$ optimization software. All computations were executed on an SGI Origin $2000^{\mathrm{TM}}$ workstation with $250 \mathrm{MHz} \mathrm{R} 10000^{\mathrm{TM}}$ processors. The (FD) sensitivity calculations were partitioned and run on several processors to reduce required memory and elapsed optimization time. This partitioning, however, results in additional accumulated computation time due to the nature of ADIFOR-generated sensitivity analysis code.

\section{Results}

The optimization results shown in this work are for design problems involving only four or eight design variables out of the 21 available wing parameters. The results shown by these authors in an earlier work:" used design variables that directly affected either the aerodynamic analysis alone or both the aerodynamic analysis and the structural analysis. In this work. additional design variables are chosen that directly affect only the structural analysis. The flow conditions for the wing optimizations were $\mu_{\curvearrowright}=0.8$ and $a=1^{\circ}$.

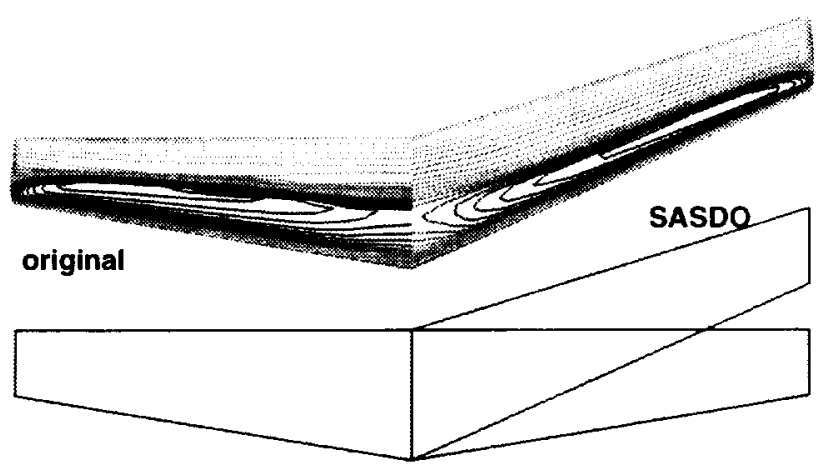

Fig. 4 Comparison of planform shapes and surface pressure contours for 4-design-variable cases, $M_{\alpha}=0.8, \alpha=1^{\circ}$.

\section{Four-Design-Variable Problems}

Table 1 and Fig. 4 show results of several optimization problems involving four design variables: the tip chord $c_{t}$, the tip setback $x_{t}$, and the structural element size factor for the two most inboard zones, $\Gamma_{1}$ and $\Gamma_{2}$. Two of the cases, designated (onv ${ }_{C}$ and SASDOC in Table 1, represent direct comparisons of SASDO and the conventional method for consistent accuracy 
Table 1 Summary of Four-Design-Variable Cases.

\begin{tabular}{l|r|rrrr} 
& baseline & \multicolumn{4}{|c}{ Optinizations } \\
& & SASDO $_{\mathrm{A}}$ & Conv $_{\mathrm{I}}$ & SASDO $_{\mathrm{C}}$ & Conv $_{\mathrm{C}}$ \\
\hline$r_{\mathrm{t}}$ & 1. & 1.07778 & 1.07281 & 1.07827 & 1.07697 \\
$\mathrm{x}_{\mathrm{t}}$ & 1. & 1.93259 & 1.91880 & 1.93052 & 1.92614 \\
$\Gamma_{1}$ & 1. & 1.06631 & 1.08008 & 1.09637 & 1.09982 \\
$\Gamma_{2}$ & 1. & 0.69510 & 0.69187 & 0.68664 & 0.68691 \\
$\mathrm{~F}$ & & & & & \\
$\mathrm{~g}_{1}$ & -7.149 & -10.187 & -10.181 & -10.186 & -10.18 .7 \\
$\mathrm{~g}_{2}$ & -.0302 & -.000760 & -.00177 & -.0000629 & -.000915 \\
$\mathrm{~g}_{3}$ & -.8882 & -0.880 & -0.876 & -0.880 & -0.879 \\
& -.2647 & -.000639 & -.000159 & -.000149 & +.000108 \\
$\varepsilon$ & & & & & \\
$\sum$ state & $9.6 \mathrm{e}-7$ & $9.9 \mathrm{e}-5$ & $8.9 \mathrm{e}-7$ & $6.6 \mathrm{e}-6$ & $4.0 \mathrm{e}-6$ \\
Egrad & 1 & 1.1 & 4.8 & 2.0 & 3.9 \\
\hline
\end{tabular}

of function and gradient analyses. The other two cases show effects of changes in the accuracy of the function and gradient analyses. The resulting designs are essentially identical for all four eases. Figure 4 show a comparison of the wing planform and the surface pressure coefficient results for the baseline design and the design designated SASDOC in Table 1 . The shock wave has been weakened somewhat in the optimized cases from that on the original wing. as one would expect. As one can sce qualitatively in Fig. 4 and unmerically from the values of the objective function $F$, the constraints $g_{i}$, and the final design variables in Table 1, the final designs are very similar for the four problems. The relative computational costs of

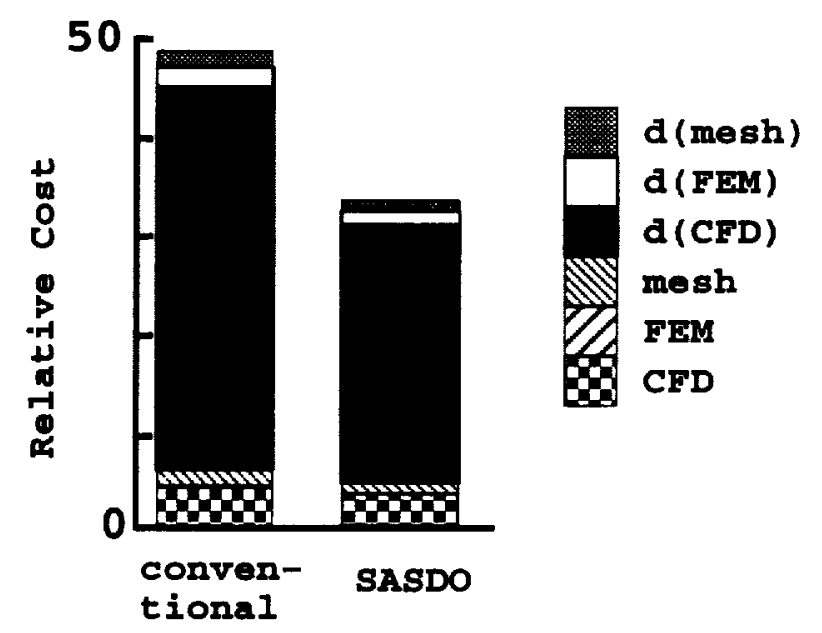

Fig. 5 Comparison of computation $\cos t$ of fourdesign-variable optimization problem using the conventional and SASDO methods.

the optimizations are slown in Table 1 and Fig. 5. The accumulated function and gradient analysis times (denoted as Estate and Egrad) are shown separately. They have been normalized by the cost of the baseline coupled function analysis. The components of the analyses were lumped together in Table 1 but they have been separated in Fig. 5. The total time for performing this optimization problem was reduced by 36 percent using the SASDO nethod. The analysis alone was reduced by 55 percent, but the gradient evaluation was the dominant cost.

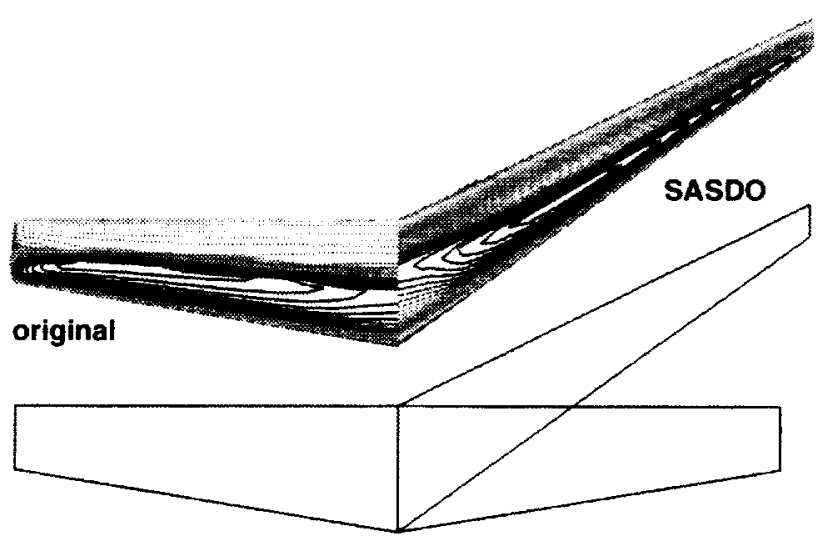

Fig. 6 Comparison of planform shapes and surface pressure contours for 8-design-variable cases, $M_{\mathrm{x}}=0.8, \alpha=1^{\circ}$.

\section{Eight-Design-Variable Problems}

Table 2 and Fig. 6 show results of three optimization problems involving eight design variables: the same set used in the four-design-variable cases with the inclusion of the span $b$, the root section max camber $z_{r}$, and the structural element size factor for two more zones, $\Gamma_{3}$ and $\Gamma_{4}$. Two of the cases, designated Conv ${ }_{B}$ and $S_{A S D O}$ in Table 2, represent direct comparisons of SASDO and the conventional method for consistent accuracy of the function and gradient analyses. Figure 6 shows a comparison of wing planform and surface pressure coefficient results for the baseline design and the design designated $\mathrm{SASDO}_{\mathrm{B}}$ in Table 2. The relative computational costs of the optimizations are shown in Table 2 and Fig. 7 . The total time for performing this optimization problem was reduced by 
Table 2 Summary of Eight-Design-Variable Cases.

\begin{tabular}{l|r|rrr} 
& baseline & \multicolumn{3}{|c}{ Optimizations } \\
& & Conv $_{\mathrm{A}}$ & SASDO $_{\mathrm{B}}$ & Conv \\
\hline $\mathrm{c}_{\mathrm{t}}$ & 1. & 0.76574 & 0.75929 & 0.75794 \\
$\mathrm{x}_{\mathrm{t}}$ & 1. & 2.60000 & 2.60000 & 2.60000 \\
$\mathrm{~b}$ & 1. & 1.05992 & 1.07543 & 1.07269 \\
$\mathrm{z}_{\mathrm{r}}$ & 1. & 1.11511 & 1.11173 & 1.11175 \\
$\Gamma_{1}$ & 1. & 2.42444 & 2.55263 & 2.44498 \\
$\Gamma_{2}$ & 1. & 2.09537 & 2.09924 & 2.09394 \\
$\Gamma_{3}$ & 1. & 1.03635 & 1.01883 & 1.05778 \\
$\Gamma_{4}$ & 1. & 0.65137 & 0.81046 & 0.76612 \\
& & & & \\
$\mathrm{~F}$ & -7.149 & -21.255 & -21.235 & -21.244 \\
$\mathrm{~g}_{1}$ & -.0302 & -.7536 & -.7255 & -.7316 \\
$\mathrm{~g}_{2}$ & -.8882 & -.00336 & -.00671 & -.000241 \\
$\mathrm{~g}_{3}$ & -.2647 & -.000420 & -.000962 & -.000134 \\
& & & & \\
$E$ & $9.6 \mathrm{e}-\bar{\tau}$ & $6.8 \mathrm{e}-7$ & $1.8 \mathrm{e}-5$ & $1.6 \mathrm{e}-5$ \\
$\sum$ state & 1 & 13.5 & 4.4 & 11.3 \\
$\sum$ grad & & 146.7 & 115.9 & 148.6
\end{tabular}

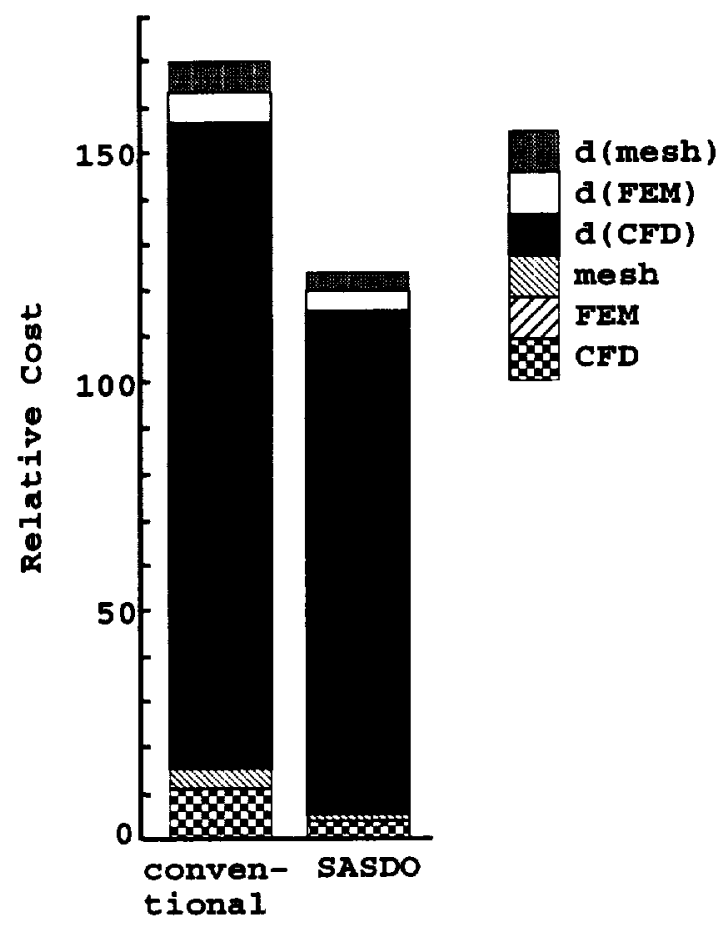

Fig. 7 Comparison of computation cost of eightdesign-variable optimization problem using the conventional and SASDO methods.

26 percent using the SASDO method. The analysis alone was reduced by 60 percent, but, as with the fourdesign-variable problem, the gradient evaluation was the dominant cost.

\section{Further Discussion}

The relative costs, based on CPI timing ratios. for the SASDO (SAND) procedures applied to these present small 3-D aerodynamic/structural design opti- mization problems are about seven-tenths of the costs of the corresponding conventional (NAND)procedures. This range is very similar to that reported for $2-D$ nonlinear aerodynamic shape design optimization in Refs. 1 and 4, even though many of the computational details differ. The results given in Ref. 1 were for a turbulent transonic flow with shock waves computed using a Navier-Stokes code; a direct differentiation approach (using ADIFOR) was used for the sensitivity analysis. The results reported in Ref. 4 were for a compressible flow without shock waves computed using a nonlinear potential flow code; an adjoint approach was used for the sensitivity analysis. Since these two optimization problems were also not the same, no timing comparison between these adjoint and direct differentiation solution approaches would be meaningful. As indicated earlier, an expected speed-up for using an adjoint approach instead of the direct differentiation approach was estimated in Ref. 1. Chattas and Bark $^{23}$ recently reported $2-D$ and $3-D$ results for optimal control of steady incompressible Navier-Stokes flow that demonstrate an order-of-magnitude reduction of (CPU time for a SAND approach versus a NAND approach. These results were obtained using reduced Hessian SQP methods that avoid converging the flow equations at each optimization iteration. The relationship of these methods with respect to other optimization techniques is also discussed in Ref. 26. The "( Control Theory" approach of Jameson ${ }^{29}$ and several other SAND-like methods for simultaneous analysis and design. which were summarized and discussed by Ta asan. "'? have been applied to aerodynamic shape design problems at several fidelities of (CFD approximation. These techniques have obtained an aerodynamic design in the equivalent of several analysis CPI times for some sample problems.

\section{Concluding Remarks}

This study has introduced an implementation of the SASDO technique for a simple, isolated wing. Initial results indicate that $S A S D O$

1. is feasible under dual simultaneity (i.e. simultaneity not only with respect to analysis and design optimization. but also simultaneity with respect to flexible wing aero-structural interaction)

2. finds the same local minimum as a conventional technique

3 . is computationally more efficient than a conventional gradient-based optimization technicue

4. requires few modifications to the analysis and sensitivity analysis codes involved

5. is effective at reducing the function analysis cost, but the gradient analysis time is the dominant $\cos t$ 


\section{Acknowledgment}

The second author, G. J.-W. Hou, was supported in this work by NASA through several Tasks under contract NAS1-19858 and NASA P.O. No. L-9291 with the Old Dominion University Research Foundation.

\section{References}

${ }^{1}$ Newman. P. A.. Hou, G. J.-W., and Taylor, III, A. C., "Observations Regarding Use of Advanced CFD Analysis, Sensitivity Analysis, and Design Codes in MDO," Multidisciplinary Design Optimization: State of the Art, edited by N. M. Alexandrov and M. Y. Hussaini. SIAM Proceedings Series, Philadelphia, 1997. pp. 263 279, also ICASE Report 96-16, NASA CR 198293, (available electronically at www.icast.edu).

${ }^{2}$ Newman, III, J. C., Taylor, III, A. C., Barnwell, R. W., Newman, P. A., and Hou, G. J.-W.. "Overview of Sensitivity Analysis and Shape Optimization for Complex Aerodynamic Configurations." Joumal of Aircraft. Vol. 36, No. 1. 1999, pp. $87-96$.

Alexandrov, N. M. and Hussaini, M. Y., editors, Multidisciplinary Design Optimization: State of the Art, SIAM Proceedings Series. Philadelphia. 1997.

${ }^{4}$ Ghat as, O. and Orozco, C. E., "A Parallel Reduced Hessian SQP Method for Shape Optimization," Maltidisciplinary Design Optimization: State of the Art, edited by N.M. Alexandrov and M. Y. Hussaini, SIAM Proceedings Series, Philadelphia. 1997. pp. 133-152.

"Hou, C. J.-W., Taylor, III, A. C., Mani, S. V., and Newman, P. A., "Simultaneous Aerodynamic: Analysis and Design Optimization." Abstracts from Ind C.S. National Congress on Computational Mechanics. Aug. 1993, pp. 130.

'Mani. S. V.. Simultaneous Aerodynamic Analysis and $D_{t}$. stgn Optimisation. Master's thesis. Old Dominion University, Norfolk, VA, Dec, 1993.

'Hou. (. J.-W.. Kurivi, V. M.. Taylor, III, A. C.. Maroju, $V$., and Newman. P. A., "Simultaneous Aerodynamic Analysis and Design Optimization (SAADO) of a Turbulent Transonic Airfoil Using a Navier-Stokes Code With Automatic: Differentiation (ADIFOR)." Proceedings of the Computational Aerosciunces Workshop 95 . edited by W. J. Feiereisen and A. K Lawer. NASA (I) CP-20010, Jan. 1996, PP. 82-85.

${ }^{8}$ Gumbert. C. R.. Hou, G. J.-W., and Newman, P. A., "Simultaneous Aerodynamic Analysis and Design Optimization (SAADO) of a 3-D Rigid Wing," Procerdings of 14 th AlAA Computational Fluid Dyrames Confererice, Norfolk. June 1999. pp. 402-418, also AIAA Paper 99-3296.

${ }^{9}$ Gumbert. ('. R., Hou, G. J.-W., and Newman, P. A., "Simultaneous Aerodynamic Analysis and Design Optimization (SAADO) of a $3-D$ Flexible Wing," ALAA Paper 2001-1107, Jan. 2001 .

${ }^{10}$ Haftka, R. T., Sobieszczanski-Sobieski, J., and Padula, S. L.. "On Options for Interdisciplinary Analysis and Design Optimization," Structural Optimization. Vol. 4, 1992, pp. 6574 .

"Shubin, G. R., "Application of Alternative Multidisciplinary Optimization Formulations to a Model Problem for Static Aeroelasticity," Journal of Computational Physics, Vul. 118, No. 1. 1995, pp. 7:3-85.

12 Arian, E.. "Analysis of the Hessian for Aeroelastic Optimization," ICASE Report 95-84, Dec. 1995.

I" Walsh, J. I.. Townsend. J. C., Salas, A. O., Samareh. J. A., Mukhopadhyay, V., and Barthelemy, J. F., "Multidisciplinary High-Fidelity Analysis and Optimization of Aerospace Vehicles, Part 1: Formulation," AIAA Paper 2000-0418, Jan. 2000.

${ }^{14}$ Walsh, J. L.. Weston, R. P., Samareh, J. A., Mason, B. H., Green, L. L., and Biedron, R. T., "Multidisciplinary HighFidelity Analysis and Optimization of Aerospace Vehicles, Part 2: Preliminary Results." AIAA Paper 2000-0419, Jan. 2000.
${ }^{15}$ Reuther, J. J., Alonso, J. J., Martins, J. R., and Smith, S. C. "A Coupled Aero-Structural Optimization Method for Complete Aircraft Configurations," AIA A Paper 99-018T. Jan. 1999.

${ }^{16}$ Kapania, R. K.. Eldred, L. B., and Barthelemy, J. -F. M., "Sensitivity Analysis of a Wing Aerwelastic Response," Journal of Aircruft, Vol. 30, No. 1, 1993. pp. 496-504, also AIAA Paper 91-1103.

${ }^{17}$ Arslan, A. E. and Carison, L. A., "Integrated Determination of Sensitivity Derivatives for an Aeroelastic Transonic Wing," Proceddings for the 5th AlAA/ISAF/NASA/ISSMO Symposium on Multidisciplinary Analysis and Optimization. Panama City Beach, FL, Sept 1994, pp. 1286-1300, also AIAA Paper 94-4400 CP.

${ }^{18}$ Giunta, A. A. and Sobieszczanski-Sobieski, J.. "Progress Toward Using Sensitivity Derivatives in a High-Ficlelity Aeroelastic: Analysis of a Supersonic Transport," Procetdings of the $7 t h$ AIAA/USAF/NASA/ISSMO Symposium on Multidisciplinary Analysis and Optimization. St. Louis, MO. Sept. 1998. Pp. 411-153, also AIAA Paper 98-4763 CP.

${ }^{19}$ Giunta, A. A., "Sensitivity Analysis for Coupled AeroStructural Systems." NASA TM-1999-209367, Aug. 1999.

${ }^{20}$ Newman, III, J. C.. Whitfield, D. L., and Anderson, K. W., "A Step-Size Independent Approach for Multidisciplinary" Sensitivity Analysis and Design Optimization," Procetdings of the 1 th Applied Aerodumamies Conference. Norfolk, VA. June 1999, pp. 12-22, also AIAA Paper 99-3101.

${ }^{21}$ Ghattas, O. and Li. X., "Domain Decomposition Methods for Sensitivity Analysis of a Nonlinear Aeroelastic Problem," $l n$ ternational Journal of Computational Fluid Dynamics. Vol. 11. 1998, pp. 113-130.

${ }^{22}$ Moller, H. and Lund. E., "Shape Sensitivity Analysis of Strongly Coupled Fluid-Structure Interaction Problems," Procedings of sth AIAA/ISAF/NASA/ISSMO Symposium on Multidisciplinary Analysis and Optimization. Long Beach. Sept. 2000, also AIAA Paper 2000-4823.

${ }^{2: 3}$ Maute, K., Nikbay, M., and Farhat, C., "Analytical Based Sensitivity Analysis and Optimization of Nonlinear Aeroelastic Systems." Proctedings of 8 th AIAA/USAF/NASA/ISSMO Symposium on Multidisciplinary Analysis and Optimization. Long Beach, Sept. 2000, also A IAA Paper 2000-4825.

${ }^{24} \mathrm{Hou}, \mathrm{G}$, and Satyanarayana, A., "Analytical Sensitivity Analysis of a Static Aeroelastic Wing," Procecdings of $8 t h$ AlAA/ISAF/NASA/JSSMO Symposium on Multidisciplinary Analysis and Optimization, Long Beach, Sept. 2000, also AIAA Paper 2000-4824.

${ }^{25}$ Biros, (1. and Ghattas, O., "Parallel Newton- Krylov Algorithms For PDE-Constrained Optimization," Proctedings of Scitntific Computing 44. Portland, OR, 1999.

${ }^{26}$ Ghattas, O, and Bark, J.-H., "Optimal Control of Twoand Three-Dimensional Navier-Stokes Flows," Journal of Caneputational Physics, Vol. 136. No. 2, 1997, pp. 231 -244.

${ }^{27}$ Pierce, N. A. and Giles, M. B., "Adjoint Recovery of Superconvergent Functionals From PDE Approximations." SlAM Revitw, Vol. 42, No. 2, 2000, pp. 247-264.

${ }^{28}$ Venditti, D. A. and Darmofal, D. L., "Multilevel Error Estimation and Grid Adaptive Strategy for Improving the Accuracy of Integral Outputs," Procetdings of the AIAA 15th Computational Fluid Dynamics Conference, Norfolk, Jume 1999. also AIAA Paper 1999-3292.

29 Jameson, A. and Vassberg. J. C.. "Computational Fluid Dynamics for Aerodynamic Design: Its Current and Future Impart." AlAA Paper 2001-0538, Jan. 2001.

${ }^{30}$ Rumsey, C.. Biedron, R., and Thomas, J., "(FI,3D): Its History and Some Recent Applications," NASA TM-112861, May 1997.

31 Sherman, L.. Taylor, III, A., Green, L., Newman, P., Hou, G., and Korivi, M., "First- and Second-Order Aerodynamic. Sensitivity Derivatives via Automatic Differentiation with Incre- 
mental Iterative Methods," Journal of Computational Physics. Vol. 129, No. 2, 1996, pp. 307-336.

${ }^{32}$ Bischof. C. H., Carle, A., Corliss, G. F., Giriewank, A., and Hovland, P., "ADIFOR: Generating Derivative Codes from Fortran Programs," Scientific Programming. Vol. 1, No. 1, 1992, pp. 1-29.

${ }^{32}$ Bischof, C. and Griewank, A., "ADIFOR: A Fortran System for Portable Automatic: Differentiation," Procetdings of the 4th AlAA/USAF/NASA/OAI Symposium on Multidisciplinary Analysis and Optimization. Cleveland. OH, Sept. 1992.pp. 433441, also AlAA Paper 92-4744 CP.

${ }^{34}$ Taylor, III. A. (.. Oloso, A., and Newman, III, J.C., "CFL3D.ADII (Version 2.0): An Efficient. Accurate. GeneralPurpose Code for Flow Shape-Sensitivity Analysis." ALAA Paper 97-2204. June 1997.

35 Smith, R. L., Bloor, M. I. G., Wilson, M. J., and Thomas, A. T., "Rapid Airplane Parametric Input Design (RAPID)," Procedings of the lath AIAA Computational Fluit Dynamics Conference, San Diego, June 1995, pp. 452-462, also AlAA Paper 95-1687.

${ }^{36}$ Jones, W. T. and Samareh-Abolhassani, J., "A Grid Generation System for Multidisciplinary Design Optimization." Procetdings of the 12th HAt Computational Find Dynamics ('onferenct. San Diego, June 1995, pp. 474-482, also AIA Paper 95-1689.

${ }^{37}$ Bischof, C.. Jones, W. T., Samareh-Abolhassani, J., and Mauer, A.. "Experiences with the Application of the ADIC Automat ic Differentiation Tool to the (SCMDO) 3-D Volume (irid Generation Code," AIAA Paper 96-0716. Jan. 1996.

${ }^{8}{ }^{N}$ guyen. D. T., "Finite Element Software for Multidisciplinary Design Optimization, Final Report," Tech. rep., Old Dominion University Research Foundation. Cont ract NAS1-19858, Task 69. NASA Langley Research Center, Hampton. VA. Nor. 1995.

${ }^{39} \mathrm{Hou}$. G., Arunkumar, S., and Tiwari. N. S., "First- and Second-Order Sensitivity Analysis of Finite Element Equations via Automatic Differentiation." Proceflings of the ith ALAA/USAF/NASA/ISSMO Symposium on Multidisciphnary Analysis and Optimization, St. Louis, MO. Sept. 1998, pp. 454464, also AIAA Paper 98-4764.

to Samareh, J. A. and Bhatia, K. G.. "A Inified Approach to Modeling Multidisciplinary Interactions." Proctedings of the 8 th AHA/NASA/USAF/ISSMO Symposium on Multidisciplinary Analysis and Optimization. Long Bearh. Sept. 2000, also AIAA Paper 2000-4704.

${ }^{11}$ Anon.. DOT lisers Manual: lersion 5.0. Vanderplaats Research \& Development, lnc., Colorado Springs, May 1999.

${ }^{12}$ Ta'asan. $\&$., "Trends in Aerodynamic Design and Optimization: A Mathematical Viewpoint." Proceedings." Proccedings of the tath ALAA Computational Flaid Dynantics Conference. San Diego, June 1995, pp. 961-970. also AIAA Paper 95-0001. 

